University of Wollongong

Research Online

Faculty of Engineering and Information

Faculty of Engineering and Information

Sciences - Papers: Part B

Sciences

2018

Deformation twinning and dislocation processes in nanotwinned copper by molecular dynamics simulations

\author{
Xing Zhao \\ University of Wollongong, xz920@uowmail.edu.au \\ Cheng Lu \\ University of Wollongong, chenglu@uow.edu.au \\ Anh Kiet Tieu \\ University of Wollongong, ktieu@uow.edu.au \\ Lihua Zhan \\ Central South University \\ Minghui Huang \\ Central South University
}

See next page for additional authors

Follow this and additional works at: https://ro.uow.edu.au/eispapers1

Part of the Engineering Commons, and the Science and Technology Studies Commons

Research Online is the open access institutional repository for the University of Wollongong. For further information contact the UOW Library: research-pubs@uow.edu.au 


\title{
Deformation twinning and dislocation processes in nanotwinned copper by molecular dynamics simulations
}

\author{
Abstract \\ Nanotwinned materials exhibit a combination of high strength and good ductility which is attributed to the \\ interactions between dislocations and twin boundaries. But no attempt has been made to explore the \\ possibility for deformation twinning in nanotwinned face-centered cubic materials. Here we use large \\ scale molecular dynamics simulations to elucidate the mechanical behaviour of nanotwinned $\mathrm{Cu}$. We \\ demonstrate that deformation twinning plays an important role in the deformation of nanotwinned $\mathrm{Cu}$ \\ with specific twin orientations, in addition to conventional dislocation slip. Deformation twins are formed \\ through the glide of Shockley partials on adjacent $\left\{\begin{array}{lll}1 & 1 & 1\end{array}\right\}$ slip planes and two twinning mechanisms are \\ identified based on the arrangement of Shockley partials. The first mechanism involves the successive \\ motion of Shockley partials of different types, named as double-Shockley partials, which forms unstable \\ thin twin plates. The second process involves the successive passage of the same twinning dislocations \\ on neighbouring slip planes, which forms stable deformation twins along one primary twinning system or \\ symmetric twinning systems. The dislocation processes involved in the dislocation-twin reactions are \\ analysed at atomic level. The orientation dependence of deformation twinning is discussed and \\ compared with available experimental results.

\section{Disciplines} \\ Engineering | Science and Technology Studies

\section{Publication Details} \\ Zhao, X., Lu, C., Tieu, A. K., Zhan, L., Huang, M., Su, L., Pei, L. \& Zhang, L. (2018). Deformation twinning and \\ dislocation processes in nanotwinned copper by molecular dynamics simulations. Computational \\ Materials Science, 142 59-71. \\ Authors \\ Xing Zhao, Cheng Lu, Anh Kiet Tieu, Lihua Zhan, Minghui Huang, Lihong Su, Linqing Pei, and Liang Zhang
}




\title{
Deformation twinning and dislocation processes in nanotwinned copper by molecular dynamics simulations
}

\author{
Xing Zhao ${ }^{\mathrm{a}, \mathrm{b}}$, Cheng $\mathrm{Lu}^{\mathrm{a}, *}$, Anh Kiet Tieu ${ }^{\mathrm{a}}$, Lihua Zhan ${ }^{\mathrm{b}}$, Minghui Huang ${ }^{\mathrm{b}}$, Lihong Su${ }^{\mathrm{a}}$, Linqing \\ Pei $^{\mathrm{a}}$, Liang Zhang ${ }^{\mathrm{a}}$ \\ ${ }^{a}$ School of Mechanical, Materials and Mechatronic Engineering, University of Wollongong, \\ Wollongong, NSW 2522, Australia \\ ${ }^{\mathrm{b}}$ State Key Laboratory of High Performance Complex Manufacturing, Central South University, \\ Changsha 410083, People's Republic of China \\ * Corresponding author. \\ E-mail address: chenglu@uow.edu.au (C. Lu).
}

\begin{abstract}
Nanotwinned materials exhibit a combination of high strength and good ductility which is attributed to the interactions between dislocations and twin boundaries. But no attempt has been made to explore the possibility for deformation twinning in nanotwinned face-centered cubic materials. Here we use large scale molecular dynamics simulations to elucidate the mechanical behaviour of nanotwinned $\mathrm{Cu}$. We demonstrate that deformation twinning plays an important role in the deformation of nanotwinned $\mathrm{Cu}$ with specific twin orientations, in addition to conventional dislocation slip. Deformation twins are formed through the glide of Shockley partials on adjacent $\{111\}$ slip planes and two twinning mechanisms are identified based on the arrangement of Shockley partials. The first mechanism involves the successive motion of Shockley partials of different types, named as double-Shockley partials, which forms unstable thin twin plates. The second process involves the successive passage of the same twinning dislocations on neighbouring
\end{abstract}


slip planes, which forms stable deformation twins along one primary twinning system or symmetric twinning systems. The dislocation processes involved in the dislocation-twin reactions are analysed at atomic level. The orientation dependence of deformation twinning is discussed and compared with available experimental results.

Keywords: Deformation twinning; Dislocation; Nanotwinned Cu; Molecular dynamics; Slip transfer

\section{Introduction}

Deformation twinning has been widely observed in coarse-grained face-centered cubic (fcc) metals and alloys, which plays an important role in plastic deformation in addition to dislocation slip [1-3]. Twinning propensity is strongly affected by stacking fault energy [4, 5]. Deformation twinning takes place easily in metals with low stacking fault energies, such as brass, $\mathrm{Cu}-\mathrm{Al}$ alloys and stainless steels; in contrast twinning hardly occurs in materials with high stacking fault energies. Deformation twinning is also promoted by high strain rates and/or low temperatures, where slip activities are restricted because the number of operable slip systems is reduced. Grain size also influences the twinning tendency in fcc metals. Twin formation is restricted with decreasing grain size. But when grain size is reduced to nanometer level, deformation twinning is greatly enhanced [6]. Metals that normally do not twin are found to twin in nanocrystalline form [7, 8]. Molecular dynamics (MD) simulation revealed extensive deformation twinning in the deformation of nanocrystalline Al [7], which was verified by later transmission electron microscopy (TEM) observations [8]. The change in twinning propensity is attributed to twin nucleation from grain boundaries [9]. The role of grain boundaries needs to be incorporated to develop new models for deformation twinning [10]. 
A number of models have been proposed to rationalize the formation of deformation twins in fcc materials $[1,11,12]$. Twin nucleation involves two essential processes: the nucleation of twin embryos and their subsequent growth to large twins [1]. It is assumed that the formation of twins requires dislocations and the twinning source can be categorized into two types: prismatic and glide [13]. In prismatic models, the primary slip does not lie on the twinning plane. Cottrell and Bilby [14] proposed the pole mechanism which involved the dissociation of a full dislocation into a Shockley partial and a sessile Frank dislocation. But only monolayer stacking fault was produced. Venables [15] extended the pole mechanism and explained how mechanical twins grew. Cohen and Weertman [16] suggested that the overlap of stacking faults formed imperfect deformation twins. These stacking faults resulted from glide of Shockley partials dissociated from piling-up dislocations near Lomer-Cottrell locks. Fujita and Mori [17] presented a similar model involving the cross-slip of stair-rod dislocations. Mahajan and Chin [18] described a simple glide model, in which the interaction between two coplanar perfect dislocations led to the nucleation of a three-layer twin. We can see that there is a lack of consensus for twin nucleation at the moment $[12,13,19]$.

Recent studies on high manganese steels reveal that deformation twinning plays a significant role in the strain-hardening behaviour of twinning-induced plasticity (TWIP) steels [20-26]. The evolution of twin structure has been extensively characterised using advanced experimental techniques [11, 25, 27]. Ueji et al. [23] reported that deformation twinning was restricted by grain refinement and strongly depended on grain orientation. Gutierrez-Urrutia and Raabe [25] systematically distinguished three types of twin substructure at high tensile strains. A low deformation twinning activity was observed in grains oriented close to <001> directions; twin substructure was developed along one primary twinning system in grains oriented between <001> 
and <111> directions; and a multiple twin substructure was built up along the primary and secondary twinning system in grains close to $<111>$ directions. Karaman et al. [28] introduced a new dislocation model to explain the formation of extrinsic stacking faults and deformation twins in theoretically untwinning directions, such as $<001>$ under tension and $<111>$ under compression. The nature of dislocations was identified by the advanced characterization tools, which provided evidence for earlier proposed twinning mechanisms [11, 27]. Recently Casillas et al. [27] reported that extrinsic stacking faults served as twin nuclei in TWIP steels using aberration corrected scanning TEM. However, it is difficult to obtain the detailed evolution of dislocation structures based on TEM studies. Little is known about the specific deformation mechanisms involved in deformation twinning.

Nanotwinned materials exhibit simultaneous ultrahigh strength and increased ductility, as well as high electrical conductivity [29, 30], which make them attractive for numerous applications. However, no attempt has been made to explore the possibility for deformation twinning in nanotwinned fcc materials until now, because twin formation cannot be controlled in nanotwinned materials despite that multiple twin structures have been extensively observed in twin-free materials [25]. Moreover, it is not easy to distinguish deformation twins and growth twins in experiments, which are identical from a crystallographic view. In this study, we use large scale MD simulations to elucidate the mechanical behaviour of nanotwinned $\mathrm{Cu}$. Our simulations reveal that deformation twinning occurs in the deformation of nanotwinned $\mathrm{Cu}$ with specific twin orientations. The atomistic mechanisms involved in the formation of deformation twins and the dislocation-twin reactions are analysed in detail. 


\section{Methods}

The simulation cells contained parallel growth twin boundaryies (TBs) with different orientations. The nanotwinned structure was constructed by continuous atomic displacements on adjacent (111) planes to get a symmetric twin while keeping the matrix unchanged [31]. Atoms above one (111) plane were shifted along [11̄] direction by the magnitude of a partial dislocation, which was repeated to produce a specific twin spacing. Then the nanotwinned model was rotated about the $[1 \overline{1} 0]$ direction to obtain samples with different orientations. The sample dimensions are around $100 \mathrm{~nm} \times 50 \mathrm{~nm} \times 2 \mathrm{~nm}$ and the total atom number is approximately 850,000 . Fig. 1 shows the cross-sectional view of fabricated samples T4A19, T4A75 and T4A90. The letters T and A in the sample names indicate the twin spacing and inclination angle.

MD simulations were performed using the large-scale parallel molecular dynamics package LAMMPS [32]. The embedded atom method potential for $\mathrm{Cu}$ by Mishin et al. [33] was used to describe the atomic interactions between $\mathrm{Cu}$ atoms. This potential was fit to experimental data and ab initio calculations and accurately reproduced many material properties, including the stacking fault energy that is important for deformation simulations. Periodic boundary conditions were imposed in all three directions to model the behaviour of bulk materials. The samples were first equilibrated at $300 \mathrm{~K}$ for $100 \mathrm{ps}$, using a Nose-Hoover thermostat and a Parrinello-Rahman barostat (an NPT ensemble). The tensile deformation was applied by continuously scaling the atomic coordinates and box size along the $X$ direction in Fig. 1 at a constant strain rate of $5 \times 10^{8}$ $\mathrm{s}^{-1}$, while along the other two directions, stresses were kept at zero using an NPT ensemble.

The atomic configurations were visualized using the scientific software package Open Visualization Tool (OVITO) [34]. Common neighbour analysis (CNA) was used to visualize the defects. Three types of atoms are defined: grey represents perfect fcc atoms, red stands for 
hexagonal close-packed (hcp) atoms (corresponding to stacking faults and TBs), and green indicates defected atoms (corresponding to GBs, dislocation cores, and other defects). Moreover, the relative shear strain of each atom with respect to its original position was calculated (embedded in OVITO) to trace the evolution of dislocation structures. Perfect fcc atoms that are not involved in the plastic deformation were eliminated for the sake of clarity in the analysis of the dislocation structures [35]. This procedure was carried out by deleting atoms with shear strain of less than 0.15 , as calculated by OVITO. In this way, the atoms that experience slip and still retain the fcc structure are coloured in grey. The dislocations were characterized using dislocation extraction algorithm Crystal Analysis [36].

The slip system in nanotwinned fcc materials can be described by the double Thompson tetrahedron $[1,6,37]$ as shown in Fig. 2a. Twin boundary is shared by the twin and the matrix. The lower Thompson tetrahedron represents matrix slip systems, while the symmetric upper tetrahedron shows twin slip systems. Although the slip directions on the twin plane are common, the slip planes $A B C(d)$ or (111) plane in the matrix and $A B C\left(d^{\prime}\right)$ or $(\overline{1} \overline{1} \overline{1})$ plane in the twin are opposite. From the cross-sectional view (Fig. 2b), the slip activities can be catalogued into three groups: dislocations on slip planes (d) or (d') are parallel to the twin plane; dislocations on slip planes (c) or (c') are inclined $70.5^{\circ}$ to twin plane; and dislocations on other slip planes (a), (b), $(001)$ or $\left(a^{\prime}\right),\left(b^{\prime}\right),(001)^{T}$ are inclined $54.7^{\circ}$ to twin plane. The nature of the dislocations was in agreement with the results from Crystal Analysis. In this study, the dislocation reactions are represented by Burgers vector equations expressed in terms of the double Thompson tetrahedron, and the same equation in vector form is also given [1]. Indices relative to the twin lattice are given the superscript $T$. The conservation of Burgers vector is fulfilled for all the dislocation reactions. 


\section{Results}

\subsection{General observation}

Fig. 3 shows three typical atomic configurations after tensile deformation, where deformation twinning occurs in addition to dislocation slip. In sample T4A19 (Fig. 3a), the dislocation processes dominated by slip transmission are accompanied by a small amount of deformation twinning. Mechanical twins are formed along one twinning system in the twinned grains, while slip activities dominate the plastic deformation in the matrix grains. These twins are only several layers of atoms thick and are embedded in the twinned grains, in contact with the growth TBs. Moreover, the thin twin plates are not stable and can be eliminated in further deformation. But most of the growth TBs retain their original coherency even after a considerable deformation.

In sample T4A75 (Fig. 3b), the twin substructure is developed along one primary twinning system in the matrix grains. These twinning dislocations continuously transmit across the growth TBs, forming parallel shear bands across the sample. In sample T4A90 (Fig. 3c), a multiple twin substructure is built up along symmetric twinning systems in the matrix and twinned grains. Complex dislocation networks are formed and the interactions between dislocations and the growth TBs lead to the formation of intersecting slip bands. The deformation twins in these two cases are stable while the original growth TBs within the shear bands are severely destroyed. The deformation kinetics that lead to the formation of deformation twins will be analysed in detail in the following sections.

\subsection{Deformation twinning in sample T4A19.5}

To reveal the atomic mechanisms involved in the formation of deformation twins, a small part of the simulation cell is extracted for further analysis. Fig. 4 and Supplementary Video 1 show 
the evolution of dislocation structures in sample T4A19.5 (Region A1 in Fig. 3). The stacking sequences of $\{111\}$ slip planes during plastic deformation are indicated at the bottom of the figure. The tensile deformation was applied along the [11ํ] direction. It is well known that fcc metals twin in tension along the $<111>$ direction [13]. But in the present simulations of nanotwinned models, twinning takes place in the twinned grains and no twinning occurs in the matrix grains. Twin formation is initiated by homogenous nucleation of a partial dislocation $\boldsymbol{\gamma}^{\prime} \mathbf{B}$ in the twinned grain and its glide leaves an intrinsic stacking fault. Very quickly a second Shockley partial $\boldsymbol{\gamma}^{\prime} \mathbf{A}$ is emitted from the upper end of the stacking fault left by $\boldsymbol{\gamma}^{\prime} \mathbf{B}$, which combines with the first partial. These two Shockley partials constitute a double-Shockley partial $\boldsymbol{\gamma}^{\prime} \mathbf{A}-\boldsymbol{\gamma}^{\prime} \mathbf{B}$ with a net Burgers vector equal to $\mathbf{D}^{\prime} \boldsymbol{\gamma}^{\prime}$ according to $\boldsymbol{\gamma}^{\prime} \mathbf{B}+\boldsymbol{\gamma}^{\prime} \mathbf{A} \rightarrow \mathbf{D}^{\prime} \boldsymbol{\gamma}^{\prime}$. After their glide, an extrinsic stacking fault or a onelayer deformation twin is formed as shown in Fig. 4a.

A third partial $\boldsymbol{\gamma}^{\prime} \mathbf{A}$ is nucleated at the lower end of the stacking fault left by $\boldsymbol{\gamma}^{\prime} \mathbf{B}$ and combines with the first partial to form another double-Shockley partial (Fig. 4b). The combination of the three Shockley partials has a total Burgers vector equal to $\mathbf{D A}$ according to $\mathbf{D}^{\prime} \boldsymbol{\gamma}^{\prime}+\boldsymbol{\gamma}^{\prime} \mathbf{A} \rightarrow \mathbf{D}^{\prime} \mathbf{A}$. After the glide of the above three partials, a three-layer microtwin is formed (Fig. 4c). But this twin plate is not stable as indicated in Fig. 4d, where it is changed to four layers of hcp atoms after the glide of a trailing partial $\mathbf{D} \boldsymbol{\gamma}^{\prime}$. Meanwhile, several twin nuclei are formed in the twinned grains after the glide of double-Shockley paritals, which can further grow if more partial dislocations are nucleated on neighbouring slip planes. In a while, the motion of two more trailing partials eliminates this twin (Fig. 4e) and the original fcc structure is restored (Fig. 4f). The propensity for nucleating trailing dislocations restricts the growth of the deformation twins and only thin twin plates are formed. 
Fig. 5 an Supplementary Video 2 show the evolution of dislocation structures of Region A2 in Fig. 3, where deformation twins are formed by the agglomeration of small twin nuclei [1]. Plastic deformation is initiated by the nucleation of a partial dislocation (Fig. 5a), in a short while, three twin nuclei are formed independently as indicated by black arrows in Fig. 5b. The right two deformation twins assemble with each other to form a six-layer twin with a stacking fault being left inside the twinned region, which will be absorbed later (Fig. 5c). Then this growing deformation twin is combined with the two-layer twin on the left nucleated from the glide of a double-Shockley partial (Fig. 5d). This twin is formed very quickly within a stain of $0.1 \%$.

The glide of Shockley partials of different types cannot only lead to the formation of deformation twins, but can also contribute to shrinkage of the original growth twins as shown in Fig. 6 and Supplementary Video 3 (Region A3 in Fig. 3). This twin first grows by two atomic layers due to the TB migration involving the motion of two twinning dislocations $\mathbf{\delta} \mathbf{C}$ as shown in Fig. 6a. A Shockley partial $\boldsymbol{\delta}^{\prime} \mathbf{B}$ is emitted from the deposited dislocation on the TB and its glide reduces the twin thickness by one atomic layer. Then a second partial dislocation $\boldsymbol{\delta}^{\prime} \mathbf{A}$ is nucleated on the same plane and the twin is also shrunken by one atomic layer. The coplanar dislocations cannot glide on the same plane and the first partial climbs downwards and its slip further thins the twin. The thickness of this twin is further reduced through the nucleation and propagation of two more dislocations $\boldsymbol{\delta}^{\prime} \mathbf{A}$ (Fig. 6c) and $\boldsymbol{\delta}^{\prime} \mathbf{B}$ (Fig. 6d). Although the two dislocations are not combined to form double-Shockley partial, the atomistic mechanism leads to the shrinkage of growth twins is similar with the twinning process in the twinned grains.

The original growth TBs restrict the propagation of the newly formed twins; hence twinning partials are piling up on the TBs. A high stress concentration is induced and accommodating slip is required to release this stress [1]. The twinning partials $\left(\gamma^{\prime} \mathbf{A}\right.$ or $\left.\gamma^{\prime} \mathbf{B}\right)$ that forms double-Shockley 
partial meet the $\mathrm{TB}$ at $30^{\circ}$ with respect to the $\langle 110\rangle$ lines and their glide produces a step with a height equal to $1 / 3$ of the $\{111\}$ interplanar spacing on the TB. Therefore, groups of three or more twinning dislocations can give steps larger than a unit height. Fig. 7 and Supplementary Video 4 show the reaction between twinning dislocations and the TBs of Region A4 in Fig. 3. At first a double-Shockley partial $\boldsymbol{\gamma}^{\prime} \mathbf{A}-\boldsymbol{\gamma}^{\prime} \mathbf{B}$ with a net Burgers vector of $\mathbf{D}^{\prime} \boldsymbol{\gamma}^{\prime}$ is deposited on the lower TB (Fig. 7a). A third partial $\gamma^{\prime} \mathbf{B}$ is nucleated and another $\mathbf{D}^{\prime} \boldsymbol{\gamma}^{\prime}$ is deposited on the upper TB (Fig. 7b). Then the forth partial dislocation $\boldsymbol{\gamma}^{\prime} \mathbf{A}$ is nucleated and combines with the deposited dislocation to form a full dislocation $\mathbf{D}^{\prime} \mathbf{A}$ on the TB. This dislocation quickly transmits across the TB and a Lomer dislocation $\mathbf{C D}$ is emitted in the matrix grain, while leaving a partial dislocation $\mathbf{B} \boldsymbol{\delta}$ on the TB (Fig. 7c). This transmission process can be expressed as:

$$
D^{\prime} A_{(c \prime)} \rightarrow C D_{(001)}+B \delta_{(d)}
$$

Or in vector form:

$$
\frac{1}{6}[\overline{1} \overline{4} \overline{1}]_{(\overline{11} 5)} \rightarrow \frac{1}{2}[\overline{1} \overline{1} 0]_{(001)}+\frac{1}{6}[2 \overline{1} \overline{1}]_{(111)}
$$

with:

$$
\frac{1}{6}[\overline{1} \overline{4} \overline{1}]_{(\overline{115})}=\frac{1}{2}[101]_{(\overline{11} 1)^{T}}^{T}
$$

The left dislocation $\mathbf{B} \boldsymbol{\delta}$ interacts with the second partial $\boldsymbol{\gamma}^{\prime} \mathbf{B}$ to form a stair-rod dislocation $\gamma^{\prime} \boldsymbol{\delta}$ according to $\boldsymbol{\gamma}^{\prime} \mathbf{B}+\mathbf{B} \boldsymbol{\delta} \rightarrow \boldsymbol{\gamma}^{\prime} \boldsymbol{\delta}$. This stair-rod dislocation then combines with an emitted trailing partial $\mathbf{D}^{\prime} \boldsymbol{\gamma}$ ' to form a Frank dislocation $\mathbf{D}^{\prime} \boldsymbol{\delta}$ on the TB according to $\boldsymbol{\gamma}^{\prime} \boldsymbol{\delta}+\mathbf{D}^{\prime} \boldsymbol{\gamma}^{\prime} \rightarrow \mathbf{D}^{\prime} \boldsymbol{\delta}$ (Fig. 7d).

The Lomer dislocation glides on anomalous (001) plane in the matrix grain. Previous studies [38] revealed that the screw segment of this dislocation may cross-slip to conventional $\{111\}$ slip planes and split into two Shockley partials according to $\mathbf{C D} \rightarrow \mathbf{C} \boldsymbol{\alpha}+\boldsymbol{\alpha D}$ or $\mathbf{C D} \rightarrow \mathbf{C} \boldsymbol{\beta}+\boldsymbol{\beta D}$, since dislocation CD is also a possible slip direction on slip planes (a) or (b). But our analysis reveals that the stress state on the extended dislocation, with compressive stress on both the leading and the trailing partials (negative Schmid factors), restricts this dissociation [2]. Therefore, it is 
difficult for this dislocation to cross-slip to $\{111\}$ slip plane [37]. The Lomer dislocation glides on (001) plane until it is blocked by the TB (Fig. 7e). Then this Lomer dislocation transmits across the TB and a leading partial $\gamma^{\prime} \mathbf{A}$ is emitted in the twinned grain. In a short while, a trailing partial $\mathbf{D}^{\prime} \gamma^{\prime}$ is emitted on the same slip plane and a Shockley partial is left on the TB (Fig. 7f). An extended dislocation is formed in the twinned grain and this transmission process can be summarised as:

$$
C D_{(001)} \rightarrow \gamma^{\prime} A_{\left(c^{\prime}\right)}+D^{\prime} \gamma_{\left({ }^{\prime}\right)}+\delta B_{(d)}
$$

Or in vector form:

$$
\frac{1}{2}[\overline{1} \overline{1} 0]_{(001)} \rightarrow \frac{1}{18}[2 \overline{7} \overline{1}]_{(\overline{11} 5)}+\frac{1}{18}[\overline{5} \overline{5} \overline{2}]_{(\overline{115})}+\frac{1}{6}[\overline{2} 11]_{(111)}
$$

with

$$
\frac{1}{18}[2 \overline{7} \overline{1}]_{(\overline{11} 5)}=\frac{1}{6}[2 \overline{1} 1]_{(\overline{11} 1)^{T}}^{T} \text { and } \frac{1}{18}[\overline{5} \overline{5} \overline{2}]_{(\overline{115})}=\frac{1}{6}[112]_{(\overline{11} 1)^{T}}^{T}
$$

Reaction (2) is a reverse path of reaction (1). Dislocation transmission may also lead to the formation of deformation twins as shown in Fig. 4f.

\subsection{Deformation twinning in sample T4A75}

Fig. 8 and Supplementary Video 5 reveal the evolution of twin substructure in sample T4A75 (Region B in Fig. 3). The stacking sequences of $\{111\}$ slip planes during deformation twinning are indicated at the bottom of the figure. The slip directions and dislocation reaction diagrams are schematically illustrated in Fig. 9. In this sample, the tensile deformation was applied along the [221] direction. Plastic deformation is initiated by homogenous nucleation of a partial dislocation $\gamma \mathbf{D}$ in the matrix grain. This dislocation has a pure edge nature and its glide creates a step with a height equal to $2 / 3$ of the $\{111\}$ interplanar spacing on the TB. Under the high stress state, this dislocation can transmit across the TB to form a Lomer dislocation $\mathbf{D}^{\prime} \mathbf{C}$ in the twinned grain, which leaves a Hirth dislocation $\gamma \mathbf{C} /$ DD' $^{\prime}$ on the TB (Fig. 8a and Fig. 9b). This transmission is expressed as: 


$$
\gamma D_{(c)} \rightarrow D^{\prime} C_{(\overline{22} 1)}+\gamma C / D D^{\prime}
$$

Or in vector form:

$$
\frac{1}{6}[\overline{1} \overline{1} \overline{2}]_{(\overline{11} 1)} \rightarrow \frac{1}{6}[\overline{1} \overline{1} \overline{4}]_{(\overline{22} 1)}+\frac{1}{3}[001]
$$

with

$$
\frac{1}{6}[\overline{1} \overline{1} \overline{4}]_{(\overline{22} 1)}=\frac{1}{2}[110]_{(001)^{T}}^{T}
$$

The motion of the Lomer dislocation produces a unit height step. The discrepancy between the perpendicular displacements across the TB results in the nucleation of two more Shockley partials $\gamma \mathbf{D}$ from the intersections between the stacking fault and the TBs as the plastic strain increases. The second dislocation from the right intersection interacts with the residual Hirth dislocation to form a stair-rod dislocation $\boldsymbol{\delta} \gamma$ on the TB (Fig. 8b) and a two-layer twin is formed after its glide. This combination can be expressed as:

$$
\gamma C / D D^{\prime}+\gamma D_{(c)} \rightarrow \delta \gamma
$$

Or in vector form:

$$
\frac{1}{3}[001]+\frac{1}{6}[\overline{1} \overline{1} \overline{2}]_{(\overline{11} 1)} \rightarrow \frac{1}{6}[\overline{1} \overline{1} 0]
$$

Combining Eqs (3) and (4), we have:

$$
2 \times \gamma D_{(c)} \rightarrow D^{\prime} C_{(\overline{22} 1)}+\delta \gamma
$$

Or in vector form:

$$
2 \times \frac{1}{6}[\overline{1} \overline{1} \overline{2}]_{(\overline{11} 1)} \rightarrow \frac{1}{6}[\overline{1} \overline{1} \overline{4}]_{(\overline{22} 1)}+\frac{1}{6}[\overline{1} \overline{1} 0]
$$

This interaction is schematically shown in Fig. 9c. The same combination occurs to the third twinning dislocation from the left intersection, and a three-layer twin is formed after it swipes over the matrix grain. Then this dislocation interacts with the stair-rod dislocation to form a Frank dislocation $\boldsymbol{\delta} \mathbf{D}$ on the TB (Fig. 8c). Later, the Frank dislocation transmits across the TB to emit a Lomer dislocation in the twinned grain (Fig. 8d). This reaction can be expressed as:

$$
\gamma D_{(c)}+\delta \gamma \rightarrow \delta D \rightarrow D^{\prime} \delta \rightarrow D^{\prime} C_{(\overline{22} 1)}+C \delta_{(d)}
$$


Or in vector form:

$$
\frac{1}{6}[\overline{1} \overline{1} \overline{2}]_{(\overline{11} 1)}+\frac{1}{6}[\overline{1} \overline{1} 0] \rightarrow \frac{1}{3}[\overline{1} \overline{1} 1] \rightarrow \frac{1}{6}[\overline{1} \overline{1} \overline{4}]_{(\overline{22} 1)}+\frac{1}{6}[\overline{1} \overline{1} 2]_{(111)}
$$

Combining Eqs (3), (4) and (6), we have

$$
3 \times \gamma D_{(c)} \rightarrow 2 \times D^{\prime} C_{(\overline{22} 1)}+C \delta_{(d)}
$$

Or in vector form:

$$
3 \times \frac{1}{6}[\overline{1} \overline{1} \overline{2}]_{(\overline{11} 1)} \rightarrow 2 \times \frac{1}{6}[\overline{1} \overline{1} \overline{4}]_{(\overline{22} 1)}+\frac{1}{6}[\overline{1} \overline{1} 2]_{(111)}
$$

This interaction is schematically shown in Fig. 9d. The successive motion of three twinning partials and the motion of two Lomer dislocations create steps with equal height that is two times of the $\{111\}$ interplanar spacing. Thus, the original growth twin is sheared by two atomic layers.

As the Lomer dislocation propagates, part of the dislocation line can dissociate into a conventional Shockley partial $\mathbf{D}^{\prime} \boldsymbol{\gamma}^{\prime}$ and a Frank dislocation $\boldsymbol{\gamma}^{\prime} \mathbf{C}$ perpendicular to the slip plane according to $\mathbf{D}^{\prime} \mathbf{C} \rightarrow \mathbf{D}^{\prime} \boldsymbol{\gamma}^{\prime}+\boldsymbol{\gamma}^{\prime} \mathbf{C}$ (Fig. 9e). The Frank dislocation is absorbed after the Lomer dislocation swipes away and the Shockley partial is retained as shown in Fig. 8c. The Lomer dislocation keeps gliding on $(001)^{T}$ plane until being blocked by the TB. Then this Lomer dislocation transmits across the TB and two Shockley partials are emitted on neighbouring slip plane in the matrix grain while leaving a stair-rod dislocation on the TB (Fig. 8d). This slip transfer can be summarised as:

$$
D^{\prime} C_{(\overline{22} 1)} \rightarrow 2 \times \gamma D_{(c)}+\gamma \delta
$$

Or in vector form:

$$
\frac{1}{6}[\overline{1} \overline{1} \overline{4}]_{(\overline{22} 1)} \rightarrow 2 \times \frac{1}{6}[\overline{1} \overline{1} \overline{2}]_{(\overline{11} 1)}+\frac{1}{6}[110]
$$

Reaction (8) is the reverse path of reaction (5). The motion of the two twinning dislocations forms an extrinsic stacking fault or a two-layer twin.

The anomalous motion of Lomer dislocation on $(\overline{2} \overline{2} 1)$ or $(001)^{T}$ slip planes involves a high Peierls barrier [35]. As the stress state decreases, the persistent motion is no longer possible, 
instead the Lomer dislocation splits into two partial dislocations connected by a sessile stair-rod dislocation (Fig. 9f), forming a Lomer-Cottrell lock as shown in Fig. 3b. The formation of this triad of dislocation agrees well with classical dislocation theory [39] and can be expressed as:

$$
D^{\prime} C_{(\overline{22} 1)} \rightarrow D^{\prime} \gamma_{\left(c^{\prime}\right)}^{\prime}+\gamma^{\prime} \delta+\delta C_{(d)}
$$

Or in vector form:

$$
\frac{1}{6}[\overline{1} \overline{1} \overline{4}]_{(\overline{22} 1)} \rightarrow \frac{1}{18}[\overline{5} \overline{5} \overline{2}]_{(115)}+\frac{1}{18}[\overline{1} \overline{1} \overline{4}]+\frac{1}{6}[11 \overline{2}]_{(111)}
$$

with

$$
\begin{gathered}
\frac{1}{6}[\overline{1} \overline{1} \overline{4}]_{(\overline{2} \overline{2} 1)}=\frac{1}{2}[110]_{(001)^{T}}^{T}, \frac{1}{18}[\overline{5} \overline{5} \overline{2}]_{(\overline{115})}=\frac{1}{6}[112]_{(\overline{11} 1)^{T}}^{T} \text { and } \frac{1}{18}[\overline{1} \overline{1} \overline{4}]= \\
\frac{1}{6}[110]^{T}
\end{gathered}
$$

The deformation twins further grow though the continuous nucleation and propagation of twinning dislocations on adjacent slip planes in the matrix grain (Fig. 8e). The continuous dislocation-TB interactions form a shear band across the samples. Another interesting thing revealed in Fig. $8 \mathrm{f}$ is that a two-layer twin is nucleated from the glide of a double-Shockley partial in the twinned matrix grain.

\subsection{Deformation twinning in sample T4A90}

Fig. 10 and Supplementary Video 6 show the evolution of dislocation structures in sample T4A90 (Region C in Fig. 3). Extensive deformation twinning takes place in addition to dislocation slip during the deformation. The tensile deformation was applied along the [111] direction, which indicates that the slip systems in the matrix and twinned grain are equivalent. Therefore, plastic strain is uniformly distributed, different from sample T4A75, where a primary twinning system in the matrix grain dominates the plastic deformation. Deformation twins are formed along symmetric twinning systems in the matrix and twinned grains, which involves the successive glide of twinning dislocations on adjacent slip planes. Although the stacking fault energy for $\mathrm{Cu}$ is low, 
a high density of mechanical twins significantly increases the elastic energy since the twin spacing is as small as $4 \mathrm{~nm}$, which supresses the growth of mechanical twins. Deformation twins may be eliminated through the glide of trailing partials and dislocation slip replaces deformation twinning.

\section{Discussion}

\subsection{Twinning mechanisms}

It is well accepted that the formation of deformation twins involves successive glide of partial dislocations on adjacent $\{111\}$ slip planes. These partial dislocations are called twinning dislocations. The question is in which way these twinning partials are arranged for twin formation [13]. Our simulation of nanotwinned $\mathrm{Cu}$ revealed two twinning mechanisms based on the arrangement of twinning dislocations. In samples with $\mathrm{TB}$ inclined $15^{\circ}$ to $30^{\circ}$ to the loading direction, the plastic deformation is dominated by the transmission of screw dislocations across the $\mathrm{TB}$ as previously reported in nanotwinned $\mathrm{Cu}[35,40-43]$. In addition, deformation twinning takes place in the twinned grains in the form of small twin plates. Twin formation involves the glide of double-Shockley partials composed of two coplanar partials of different types. After the nucleation of one partial, the other one would be emitted on neighbouring slip plane as the two partials have the same Schmid factor, instead of nucleation of a trailing dislocation, which can only be emitted after the leading partial swipes over the splitting distance of an extended dislocation due to the mutual repulsive force [44]. On the other hand, the two partials have opposite screw components that they tend to be combined to form a double-Shockley partial. This dislocation has a net Burgers vector equal to the trailing partial and doubled Schmid factor as the sum of the two composing dislocations, hence it can easily glide. But these twins are not stable as the activation of double-Shockley partial is followed by the nucleation of trailing partials that have 
doubled Schmid factors compared to the twinning dislocations, which eliminates the deformation twins. Thus, dislocation slip takes over the role of deformation mechanism from deformation twinning, which restricts the growth of deformation twins. Deformation twins are continuously formed and eliminated throughout the simulations.

The simulations of nanocrystalline Al by Yamakov et al. [7, 10] also revealed that the nucleation of double-Shockley partial led to the formation of microtwins, which agrees with present simulations. Simulations of Al under shear deformation along an inverse twinning direction revealed that deformation twins were formed through the activation of two coplanar twinning systems [45]. Karaman et al. [28] proposed that extrinsic stacking faults served as nuclei for twin formation, which was confirmed by recent in situ TEM observations [27]. The doubleShockley partial has a net Burgers vector of a single partial, which means the plastic strain is halved. In situ nanoindentation of bimetal $\mathrm{Cu} / \mathrm{Nb}$ composite revealed that a two-layer twin nucleus is formed by stacking two partial dislocations of different types [46]. The flat interface after twinning indicated that the twin growth involved a combination of twinning dislocations with zero net Burgers vector [47], which is similar to zero macroscopic strain twinning mechanism observed in nanocrystalline metals [48]. Wang et al. [49] described a similar detwinning mechanism in nanotwinned $\mathrm{Cu}$ through the collective glide of twinning dislocations that form incoherent $\mathrm{TBs}$ in sample preparation. Although deformation twinning take place in the twinned grains that are favourably oriented with the applied stress for twinning, these twins cannot cut through the TBs without a high stress level. The transmission involves nucleation of Lomer dislocations across the TB and its motion requires to overcome a high barrier. It can be expected that the transmission of dislocations across TBs, GBs or the bimetal interface is more difficult, thus the smooth TBs, GBs or flat interface is retained. As for the zero-strain twinning mechanism, the sum of plastic strain 
induced by the motion of three twinning systems on one slip plane is zero, but their Schmid factors have the same relation. Therefore, simultaneous activation of three twinning systems is difficult under normal deformation.

Deformation twins are formed through the glide of the same Shockley partials in samples with $\mathrm{TB}$ inclined $75^{\circ}$ to $90^{\circ}$ to the loading direction. In sample T4A75, only one primary twinning system in the matrix grains is activated. In sample T4A90, symmetric twinning systems in the matrix and the twinned grains are activated. There was a consensus that twins formed from dislocations because the formation of twins was preceded by dislocation slip [14]. The common characteristic of materials that twin under deformation is that the activation of twinning system is accompanied by slip systems, such as $\langle 111\rangle$ orientated crystals in tension or $<001\rangle$ oriented crystals in compression. Deformation twinning requires a higher stress level than dislocation slip, under which multiple slip can be activated. The pole mechanism $[14,15]$ involves the dissociation of full dislocations to a Frank dislocation and twinning dislocations, such as $\mathbf{C D} \rightarrow \mathbf{C} \boldsymbol{\gamma}+\boldsymbol{\gamma} \mathbf{D}$, $\mathbf{A D} \rightarrow \mathbf{A} \boldsymbol{\alpha}+\boldsymbol{\alpha} \mathbf{D}$ or $\mathbf{B D} \rightarrow \mathbf{B} \boldsymbol{\beta}+\boldsymbol{\beta D}$. In our simulations, this dissociation only occurs to Lomer dislocations (Fig. 9e), which further dissociates to form Lomer-Cottrell locks (Fig. 9f). The twinning model proposed by Mahajan and Chin [18] required the dissociation of two coplanar dislocations $\mathbf{A D}+\mathbf{B D} \rightarrow \mathbf{A} \gamma+\gamma \mathbf{D}+\mathbf{B} \gamma+\gamma \mathbf{D} \rightarrow \mathbf{3} \times \gamma \mathbf{D}$, forming a three-layer twin. Full dislocations in these models are nucleated by successive emission of leading and trailing partials on the same slip plane, with the leading partials also being the twinning partials.

The mechanical behaviour of nanotwinned metals under uniaxial strain perpendicular to the TB has been widely investigated, where stacking faults or thin twin plates were observed $[38,50-$ 52]. Simulations of Al under shear deformation along a twinning direction revealed that deformation twinning occurred through the activation of the corresponding twinning systems [45]. 
The compressive strain as low as $-0.8 \%$ along $<001>$ direction is compensated by mechanical twinning in $\mathrm{Au}$ thin films [53]. Deformation twinning have been observed in $\langle 110\rangle$ oriented nanowires in MD simulations [54], which was confirmed by later in situ TEM studies [55]. Twinning was also identified to dominate the plastic deformation in nanocrystalline $\mathrm{W}$ [56]. All these studies indicate that twinning is the main deformation mechanism without significant contribution from dislocation slip. In nanotwinned samples oriented close $<111>$ direction under tensile deformation, it is possible that only leading partials are nucleated and their continuous emission on adjacent slip planes leads to the formation of mechanical twins.

\subsection{Orientation dependence of deformation twinning}

The simulations reveal that deformation twinning is strongly dependent on the crystallographic orientation of nanotwinned fcc metals. The deformation mechanism of nanotwinned materials has been classified in terms of hard and soft modes [57]. Extensive twin migration (either twinning or detwinning) was observed in samples with slant TBs [58]. Moreover, twinning was not observed in the presence of TBs in axially twinned $\mathrm{Cu}$ [59]. In the present studies, three types of twin structure have been identified according to the crystallographic orientation, which to some extent agrees with the twin substructure in TWIP steels [20, 24, 25]. The specific twinning systems changes due to existence of the TB as more twinning systems are introduced. In sample T4A19.5, the $[11 \overline{1}]$ loading direction is close to $[22 \overline{1}]$ or $[00 \overline{1}]^{T}$ direction $\left(\mathrm{A} 35.3^{\circ}\right)$ of the twinned grains, inclined with an angle of $15.8^{\circ}$. As the inclination angle changes from $15^{\circ}$ to $30^{\circ}$, the loading direction intersects with $[00 \overline{1}]^{T}$ direction in the twinned grains at angles between $20.3^{\circ}$ and $5.3^{\circ}$. Deformation twinning takes place in the twinned grains. Similarly, as the inclination angle changes from $-15^{\circ}$ to $-30^{\circ}$, the intersecting angle between the loading direction and the [001] direction $\left(\mathrm{A}-35.3^{\circ}\right)$ in the matrix grains changes from $20.3^{\circ}$ to $5.3^{\circ}$. In these cases, deformation 
twinning is expected to occur in the matrix grains. It can be seen deformation twinning occurs in grains oriented close to the $<001>$ direction with an inclination angle between $5^{\circ}$ and $20^{\circ}$. The analysis of twin substructure in TWIP steels revealed that grains oriented close to $\langle 001\rangle$ directions within an angular range of $15^{\circ}$ were characterized by a low deformation twinning activity [24, 25], which agree well with the present simulations (A15-A30).

In sample T4A75, the loading direction [221] is $15^{\circ}$ inclined with the [111] orientation (A90). Deformation twins are developed along one twinning system in the matrix grains. Due to the structural symmetry, similar deformation twins are expected to be formed along twinning system in the twinned grains in samples A105 or A-75 close to [112] direction (A109.5). In the deformation of TWIP steels, twin substructure was built up along one primary twinning system in grains oriented between $\langle 001\rangle$ and $\langle 111\rangle$ directions [24, 25], e.g. [112] direction. These mechanical twins were formed from the activation of twinning systems on slip planes (a) and (b), which have the same projection along the [110] direction on (11̄0) plane (blue lines in Fig. 2b), hence only one sets of parallel slip lines were observed $[24,25]$. But these twinning systems are restricted in present simulations due to the columnar structure [44], consequently the primary twin structures result from different twinning mechanisms. Moreover, the orientations close to [221] directions were not stable which was significantly eliminated after deformation in experiments $[24,25]$. In situ studies are needed to obtain detailed evolution of the twin structure.

In sample T4A90, the loading axis is along the [111] direction. Deformation twins are formed along symmetric twinning systems. Moreover, dislocation processes can be seen on slip planes (a) and (b) although the corresponding slip systems are restricted. It can be expected that due to the symmetry of the three slip planes, a multiple twin structure would be formed in fully threedimensional samples. The studies on TWIP steels revealed that twin structure was built up along 
more than one twinning systems in grains close to $<111>$ orientations $[20,24,25]$, as indicated by intersecting slip lines (Fig. 2b).

\subsection{Slip-twin interactions}

Our simulations reveal that nanotwinned $\mathrm{Cu}$ can experience deformation twinning in addition to dislocation slip during deformation. The growth TBs restrict the motion of dislocations and deformation twins, which gives rise to pile-ups of dislocations. Given the dense dislocation networks, various dislocation-twin reactions take place throughout the simulations which are analysed at the atomic level. Previous analysis focused on dislocations that creates steps with heights equal to integral multiples of the unit $\{111\}$ interplanar spacing [1], where the shear strain can be fully transmitted. The motion of non-screw full dislocation creates a unit height step on the TB and this dislocation can transmit across the TB to emit a Lomer dislocation with the aid of a residual Shockley partial on the twin plane. The interactions between twinning dislocations and TBs are considerably more complex. The transmission of one or two twinning dislocations emits a Lomer dislocation, leaving a Hirth dislocation or a stair-rod dislocation respectively on the twin plane. Three twinning dislocations transmit across the TB to form two Lomer dislocations with the help of a Shockley partial on the twin plane. The nucleation of Lomer dislocation can be understood in two ways. On one hand, this dislocation experiences a high resolved shear stress, as indicated by the maximum Schmid factor. On the other hand, the $\{001\}$ slip plane intersects with the twinning planes along $\langle 110\rangle$ directions on the TB. As the stress decreases, the Lomer dislocation dissociates in the slipped region to release the elastic energy of anomalous $\{001\}$ motion. 


\section{Conclusions}

Deformation twinning plays an important role in the plastic deformation of nanotwinned $\mathrm{Cu}$ with specific crystallographic orientations. Twin formation involves the glide of Shockley partials on contiguous $\{111\}$ slip planes. Two twinning mechanisms are identified based on the arrangement of these Shockley partials. The first mechanism involves the motion of the doubleShockley partial, which results from the overlap of two Shockley partials of different types. This kind of twinning occurs when the TB is inclined by an angle between $15^{\circ}$ and $30^{\circ}$ to the loading direction. The formed thin twin plates embedded either in the twinned grains or in the matrix grain are not sable and can be easily eliminated during later stages of deformation. The second twinning process results from the passage of Shockley partials of the same type. Twinning takes place when the TB is inclined by an angle between $75^{\circ}$ and $90^{\circ}$ to the loading axis. Deformation twins are well developed along one primary twinning system in samples with inclination angles close to $75^{\circ}$, while symmetric twinning takes place both in the matrix and twinned grains in samples with inclination angles close to $90^{\circ}$. The interactions between dislocation and TBs promote twin formation. The orientation dependence of deformation twinning agrees with the experimental observations in TWIP steels to some extent.

\section{Acknowledgements}

This work was supported by the National Basic Research Program of China (grant number 2014CB046602), the Key Program of National Natural Science Foundation of China (grant number 51235010), the National Natural Science Foundation of China (grant number 51674303, 51701030) and the Open Research Fund of State Key Laboratory of High Performance Complex

Manufacturing, Central South University (grant number Kfkt2015-03). X.Z., L.P. and L.Z. 
acknowledge the financial support from the China Scholarship Council. The authors acknowledge the use of computing resources on high performance computing (HPC) cluster at University of Wollongong.

\section{References}

[1] J.W. Christian, S. Mahajan. Deformation twinning, Progress in Materials Science 39 (1995) 1-157.

[2] I.J. Beyerlein, X. Zhang, A. Misra. Growth twins and deformation twins in metals, Annual Review of Materials Research 44 (2014) 329-363.

[3] M.A. Meyers, O. Vöhringer, V.A. Lubarda. The onset of twinning in metals: A constitutive description, Acta Mater 49 (2001) 4025-4039.

[4] E. El-Danaf, S.R. Kalidindi, R.D. Doherty. Influence of grain size and stacking-fault energy on deformation twinning in fcc metals, Metallurgical and Materials Transactions A: Physical Metallurgy and Materials Science 30 (1999) 1223-1233.

[5] A. Rohatgi, K.S. Vecchio, G.T. Gray. The influence of stacking fault energy on the mechanical behavior of $\mathrm{Cu}$ and $\mathrm{Cu}-\mathrm{Al}$ alloys: Deformation twinning, work hardening, and dynamic recovery, Metallurgical and Materials Transactions A 32 (2001) 135-145.

[6] Y.T. Zhu, X.Z. Liao, X.L. Wu. Deformation twinning in nanocrystalline materials, Progress in Materials Science 57 (2012) 1-62.

[7] V. Yamakov, D. Wolf, S.R. Phillpot, A.K. Mukherjee, H. Gleiter. Dislocation processes in the deformation of nanocrystalline aluminium by molecular-dynamics simulation, Nat Mater 1 (2002) 45-48.

[8] M. Chen, E. Ma, K.J. Hemker, H. Sheng, Y. Wang, X. Cheng. Deformation twinning in nanocrystalline aluminum, Science 300 (2003) 1275-1277.

[9] X.L. Wu, Y.T. Zhu. Inverse Grain-Size Effect on Twinning in Nanocrystalline Ni, Physical Review Letters 101 (2008) 025503.

[10] V. Yamakov, D. Wolf, S.R. Phillpot, H. Gleiter. Deformation twinning in nanocrystalline Al by molecular-dynamics simulation, Acta Mater 50 (2002) 5005-5020.

[11] H. Idrissi, K. Renard, L. Ryelandt, D. Schryvers, P.J. Jacques. On the mechanism of twin formation in Fe-Mn-C TWIP steels, Acta Mater 58 (2010) 2464-2476.

[12] D.R. Steinmetz, T. Jäpel, B. Wietbrock, P. Eisenlohr, I. Gutierrez-Urrutia, A. SaeedAkbari, T. Hickel, F. Roters, D. Raabe. Revealing the strain-hardening behavior of twinninginduced plasticity steels: Theory, simulations, experiments, Acta Mater 61 (2013) 494-510.

[13] S. Mahajan. Critique of mechanisms of formation of deformation, annealing and growth twins: Face-centered cubic metals and alloys, Scripta Mater 68 (2013) 95-99.

[14] A.H. Cottrell, B.A. Bilby. A mechanism for the growth of deformation twins in crystals, Philos Mag 42 (1951) 573-581.

[15] J.A. Venables. Deformation twinning in face-centred cubic metals, Philos Mag 6 (1961) 379-396.

[16] J.B. Cohen, J. Weertman. A dislocation model for twinning in f.c.c. metals, Acta Metallurgica 11 (1963) 996-998. 
[17] H. Fujita, T. Mori. A formation mechanism of mechanical twins in F.C.C. Metals, Scripta Metallurgica 9 (1975) 631-636.

[18] S. Mahajan, G.Y. Chin. Formation of deformation twins in f.c.c. crystals, Acta Metallurgica 21 (1973) 1353-1363.

[19] Q. Yu, L. Qi, K. Chen, R.K. Mishra, J. Li, A.M. Minor. The Nanostructured Origin of Deformation Twinning, Nano Letters 12 (2012) 887-892.

[20] I. Karaman, H. Sehitoglu, K. Gall, Y.I. Chumlyakov, H.J. Maier. Deformation of single crystal hadfield steel by twinning and slip, Acta Mater 48 (2000) 1345-1359.

[21] S. Allain, J.P. Chateau, O. Bouaziz. A physical model of the twinning-induced plasticity effect in a high manganese austenitic steel, Materials Science and Engineering A 387-389 (2004) 143-147.

[22] P. Yang, Q. Xie, L. Meng, H. Ding, Z. Tang. Dependence of deformation twinning on grain orientation in a high manganese steel, Scripta Mater 55 (2006) 629-631.

[23] R. Ueji, N. Tsuchida, D. Terada, N. Tsuji, Y. Tanaka, A. Takemura, K. Kunishige. Tensile properties and twinning behavior of high manganese austenitic steel with fine-grained structure, Scripta Mater 59 (2008) 963-966.

[24] I. Gutierrez-Urrutia, S. Zaefferer, D. Raabe. The effect of grain size and grain orientation on deformation twinning in a Fe-22wt.\% Mn-0.6wt.\% C TWIP steel, Materials Science and Engineering A 527 (2010) 3552-3560.

[25] I. Gutierrez-Urrutia, D. Raabe. Dislocation and twin substructure evolution during strain hardening of an Fe-22 wt.\% Mn-0.6 wt.\% C TWIP steel observed by electron channeling contrast imaging, Acta Mater 59 (2011) 6449-6462.

[26] Y. Wei, Y. Li, L. Zhu, Y. Liu, X. Lei, G. Wang, Y. Wu, Z. Mi, J. Liu, H. Wang, H. Gao. Evading the strength-ductility trade-off dilemma in steel through gradient hierarchical nanotwins, Nat. Commun. 5 (2014) 3580.

[27] G. Casillas, A.A. Gazder, E.V. Pereloma, A.A. Saleh. Evidencing extrinsic stacking faults in twinning-induced plasticity steel, Materials Characterization 123 (2017) 275-281.

[28] I. Karaman, H. Sehitoglu, Y.I. Chumlyakov, H.J. Maier, I.V. Kireeva. Extrinsic stacking faults and twinning in Hadfield manganese steel single crystals, Scripta Mater 44 (2001) 337-343.

[29] L. Lu, Y. Shen, X. Chen, L. Qian, K. Lu. Ultrahigh Strength and High Electrical Conductivity in Copper, Science 304 (2004) 422-426.

[30] L. Lu, X. Chen, X. Huang, K. Lu. Revealing the maximum strength in nanotwinned copper, Science 323 (2009) 607-610.

[31] W.D. Callister Jr. Materials science and engineering : an introduction. seventh ed., John Wiley \& Sons, New York, 2007.

[32] S. Plimpton. Fast parallel algorithms for short-range molecular dynamics, Journal of Computational Physics 117 (1995) 1-19.

[33] Y. Mishin, M.J. Mehl, D.A. Papaconstantopoulos, A.F. Voter, J.D. Kress. Structural stability and lattice defects in copper: Ab initio, tight-binding, and embedded-atom calculations, Phys Rev B 63 (2001) 224106.

[34] A. Stukowski. Visualization and analysis of atomistic simulation data with OVITO-the Open Visualization Tool, Modelling and Simulation in Materials Science and Engineering 18 (2010) 015012.

[35] X. Zhao, C. Lu, A.K. Tieu, L. Pei, L. Zhang, K. Cheng, M. Huang. Strengthening mechanisms and dislocation processes in \&lt;111\&gt; textured nanotwinned copper, Materials Science and Engineering A 676 (2016) 474-486. 
[36] A. Stukowski, K. Albe. Extracting dislocations and non-dislocation crystal defects from atomistic simulation data, Modelling and Simulation in Materials Science and Engineering 18 (2010) 085001.

[37] J.P. Hirth, J. Lothe. Theory of Dislocations. second ed., John Wiley, New York, 1982.

[38] Z.X. Wu, Y.W. Zhang, D.J. Srolovitz. Deformation mechanisms, length scales and optimizing the mechanical properties of nanotwinned metals, Acta Mater 59 (2011) 6890-6900.

[39] J. Weertman, J.R. Weertman. Elementary Dislocation Theory, Oxford University Press, New York, 1992.

[40] X. Zhao, C. Lu, A.K. Tieu, L. Pei, L. Zhang, L. Su, L. Zhan. Deformation mechanisms in nanotwinned copper by molecular dynamics simulation, Materials Science and Engineering A 687 (2017) 343-351.

[41] Y. Morris Wang, F. Sansoz, T. Lagrange, R.T. Ott, J. Marian, T.W. Barbee, A.V. Hamza. Defective twin boundaries in nanotwinned metals, Nat Mater 12 (2013) 697-702.

[42] Z.X. Wu, Y.W. Zhang, D.J. Srolovitz. Dislocation-twin interaction mechanisms for ultrahigh strength and ductility in nanotwinned metals, Acta Mater 57 (2009) 4508-4518.

[43] H. Zhou, X. Li, S. Qu, W. Yang, H. Gao. A Jogged Dislocation Governed Strengthening Mechanism in Nanotwinned Metals, Nano Letters 14 (2014) 5075-5080.

[44] V. Yamakov, D. Wolf, M. Salazar, S.R. Phillpot, H. Gleiter. Length-scale effects in the nucleation of extended dislocations in nanocrystalline Al by molecular-dynamics simulation, Acta Mater 49 (2001) 2713-2722.

[45] N.P. Daphalapurkar, K.T. Ramesh. Orientation dependence of the nucleation and growth of partial dislocations and possible twinning mechanisms in aluminum, J Mech Phys Solids 60 (2012) 277-294.

[46] S.J. Zheng, I.J. Beyerlein, J. Wang, J.S. Carpenter, W.Z. Han, N.A. Mara. Deformation twinning mechanisms from bimetal interfaces as revealed by in situ straining in the TEM, Acta Mater 60 (2012) 5858-5866.

[47] S. Zheng, I.J. Beyerlein, J.S. Carpenter, K. Kang, J. Wang, W. Han, N.A. Mara. Highstrength and thermally stable bulk nanolayered composites due to twin-induced interfaces, Nat. Commun. 4 (2013) 1696.

[48] X.L. Wu, X.Z. Liao, S.G. Srinivasan, F. Zhou, E.J. Lavernia, R.Z. Valiev, Y.T. Zhu. New Deformation Twinning Mechanism Generates Zero Macroscopic Strain in Nanocrystalline Metals, Physical Review Letters 100 (2008) 095701.

[49] J. Wang, N. Li, O. Anderoglu, X. Zhang, A. Misra, J.Y. Huang, J.P. Hirth. Detwinning mechanisms for growth twins in face-centered cubic metals, Acta Mater 58 (2010) 2262-2270.

[50] D. Jang, X. Li, H. Gao, J.R. Greer. Deformation mechanisms in nanotwinned metal nanopillars, Nature Nanotechnology 7 (2012) 594-601.

[51] J. Wang, F. Sansoz, J. Huang, Y. Liu, S. Sun, Z. Zhang, S.X. Mao. Near-ideal theoretical strength in gold nanowires containing angstrom scale twins, Nat. Commun. 4 (2013) 1742.

[52] L. Pei, C. Lu, X. Zhao, L. Zhang, K. Cheng, G. Michal, K. Tieu. Brittle versus ductile behaviour of nanotwinned copper: A molecular dynamics study, Acta Mater 89 (2015) 1-13.

[53] G. Dehm, S.H. Oh, P. Gruber, M. Legros, F.D. Fischer. Strain compensation by twinning in Au thin films: Experiment and model, Acta Mater 55 (2007) 6659-6665.

[54] H.S. Park, K. Gall, J.A. Zimmerman. Deformation of FCC nanowires by twinning and slip, J Mech Phys Solids 54 (2006) 1862-1881. 
[55] S. Lee, J. Im, Y. Yoo, E. Bitzek, D. Kiener, G. Richter, B. Kim, S.H. Oh. Reversible cyclic deformation mechanism of gold nanowires by twinning-detwinning transition evidenced from in situ TEM, Nat. Commun. 5 (2014) 3033.

[56] J. Wang, Z. Zeng, C.R. Weinberger, Z. Zhang, T. Zhu, S.X. Mao. In situ atomic-scale observation of twinning-dominated deformation in nanoscale body-centred cubic tungsten, Nat Mater 14 (2015) 594-600.

[57] T. Zhu, H. Gao. Plastic deformation mechanism in nanotwinned metals: An insight from molecular dynamics and mechanistic modeling, Scripta Mater 66 (2012) 843-848.

[58] Y. Wei. Anisotropic size effect in strength in coherent nanowires with tilted twins, Phys Rev B 84 (2011) 014107.

[59] G. Sainath, B.K. Choudhary. Molecular dynamics simulation of twin boundary effect on deformation of Cu nanopillars, Physics Letters A 379 (2015) 1902-1905. 


\section{Figures}
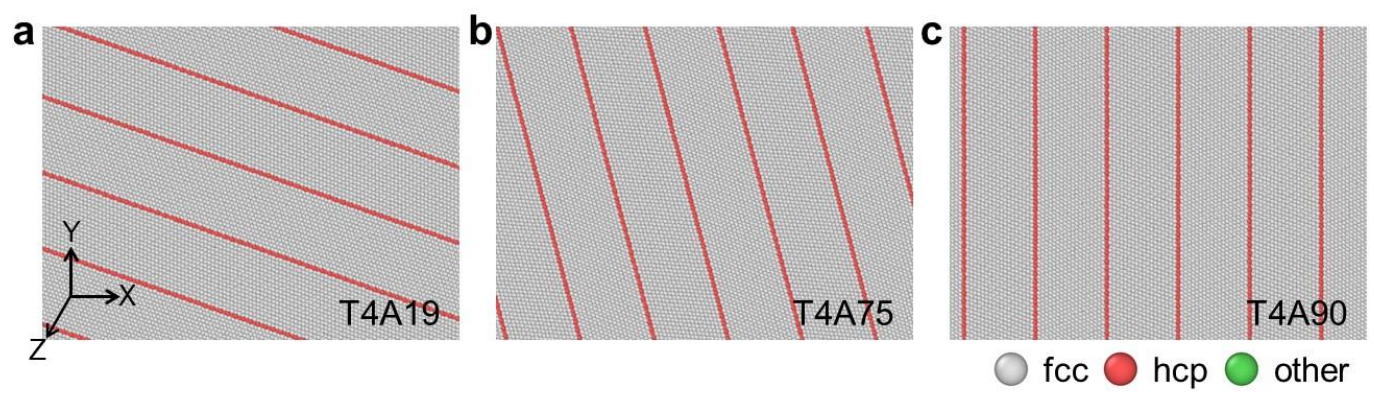

Fig. 1. Cross-sectional view of constructed nanotwinned samples with different orientation: T4A19 with [11̄]] orientation (a), T4A75 with [221] orientation (b), T4A90 with [111] orientation (c).

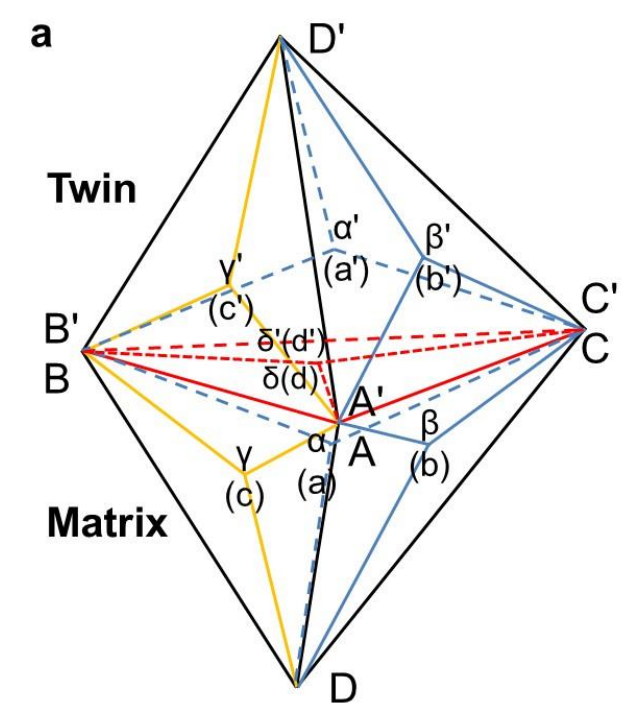

b

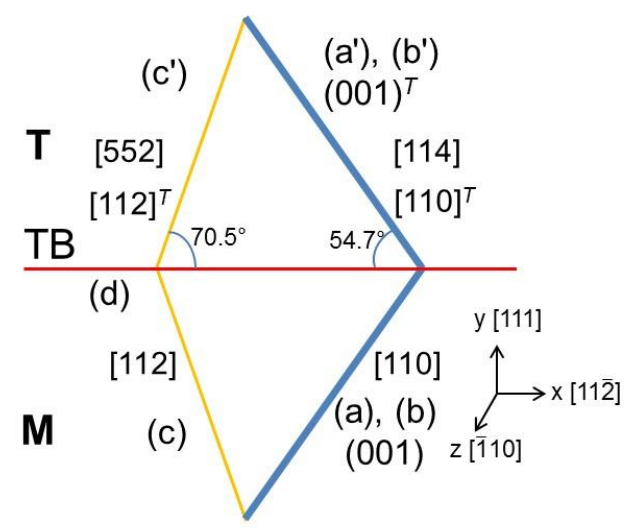

Fig. 2. Slip systems in nanotwinned fcc materials. (a) Double Thompson tetrahedron. Dislocation activities on symmetric slip planes are indicated by the same colour. (b) Cross-sectional view of dislocation activities along [1ํㅣㄹ direction. Three distinct dislocation activities on different slip planes are identified. 

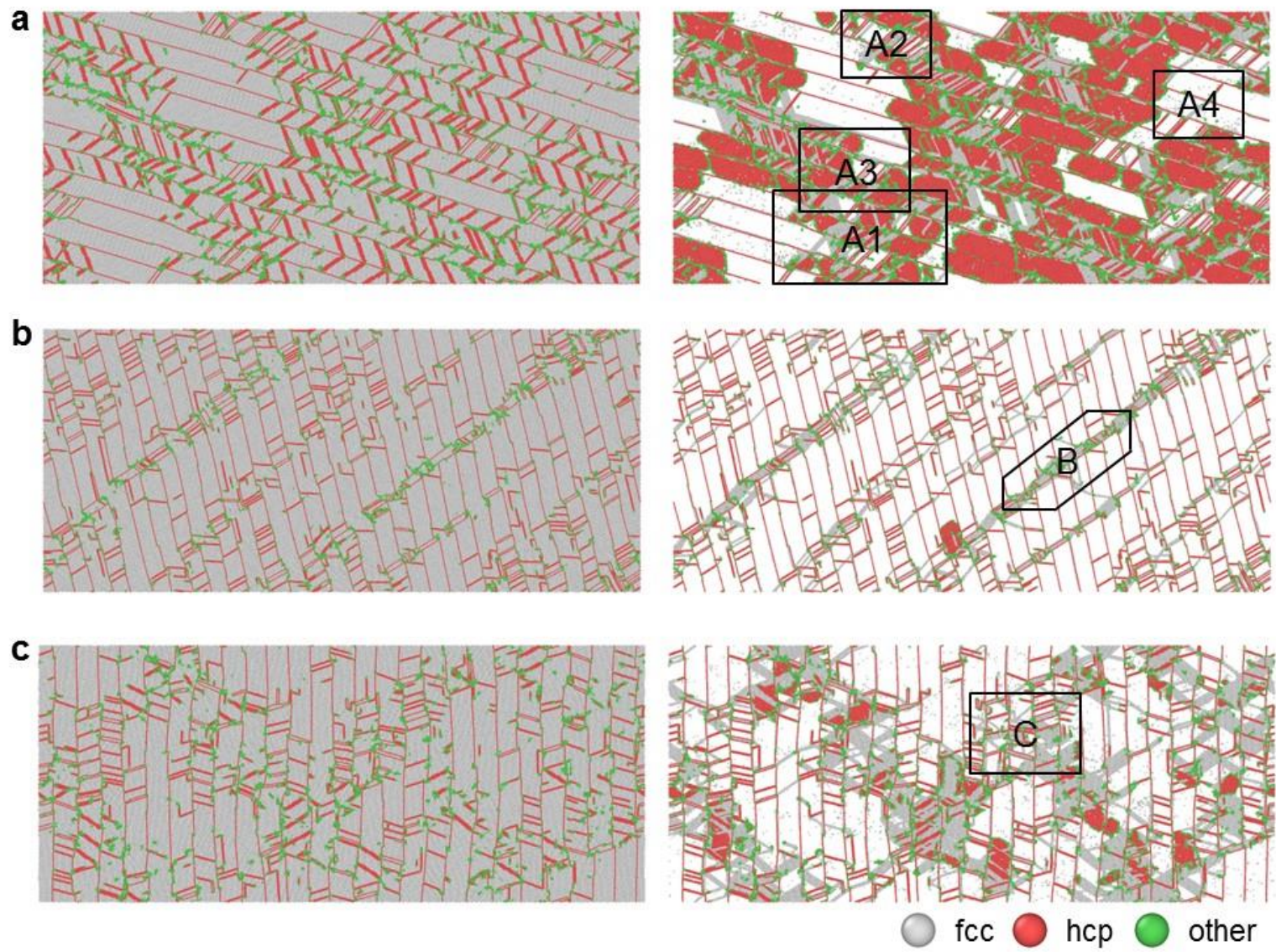

Fig. 3. Typical deformation structures containing deformation twins. (a) Thin twin plates in sample T4A19.5 at 9.3\% strain, (b) Deformation twins along one primary twinning system in sample T4A75 at 7.5\% strain (c) Deformation twins along symmetric twinning systems in sample T4A90 at $9 \%$ strain. In the right figures, perfect fcc atoms that are not involved in the plastic deformation are eliminated for clarity. 

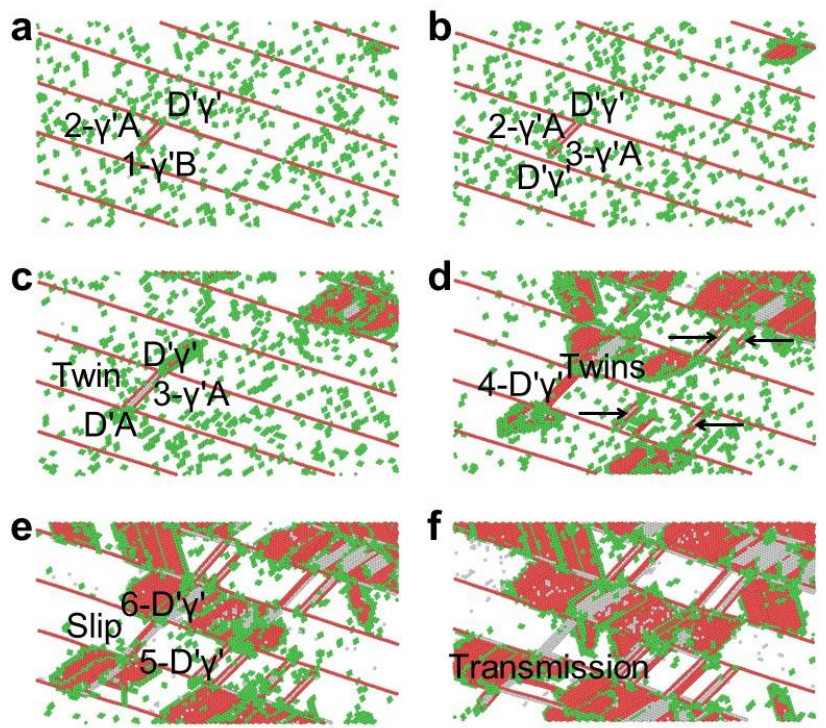

fcc hcp other

\section{0.}

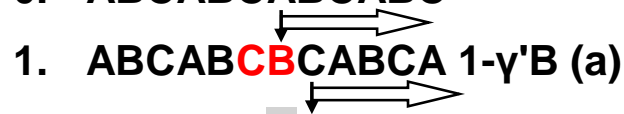

2. $A B C A B C B A B C A B$ 2- $y^{\prime} A(a / b)$

3. CABCACBABCAB 3-v'A (c)

4. CABCACACABCA 4-D'Y' (d)

5. CABCACABCABC 5-D'Y' (e)

6. $\overbrace{A B C A B C A B C A B C} 6-D^{\prime} Y^{\prime}(e / f)$

Fig. 4. Deformation twinning in sample T4A19.5 (Region A1 in Fig. 3). (a) The nucleation of a two-layer twin through the glide of a double-Shockley partial at $8.6 \%$ strain. (b) The nucleation of another double-Shockley partial at $8.63 \%$ strain. (c) The formation of a three-layer twin at $8.7 \%$ strain. (d) The three-layer twin is changed to four layers of hep atoms after the glide of a trailing partial and several deformation twins are formed at $8.78 \%$ strain. (e) Glides of two more trailing partials eliminate this twin at $8.84 \%$ strain. (f) Dislocation structure at $8.94 \%$ strain. The stacking sequences during deformation are shown below the figure. The red letters indicate TB and the twin lamella are highlighted by grey colour. 

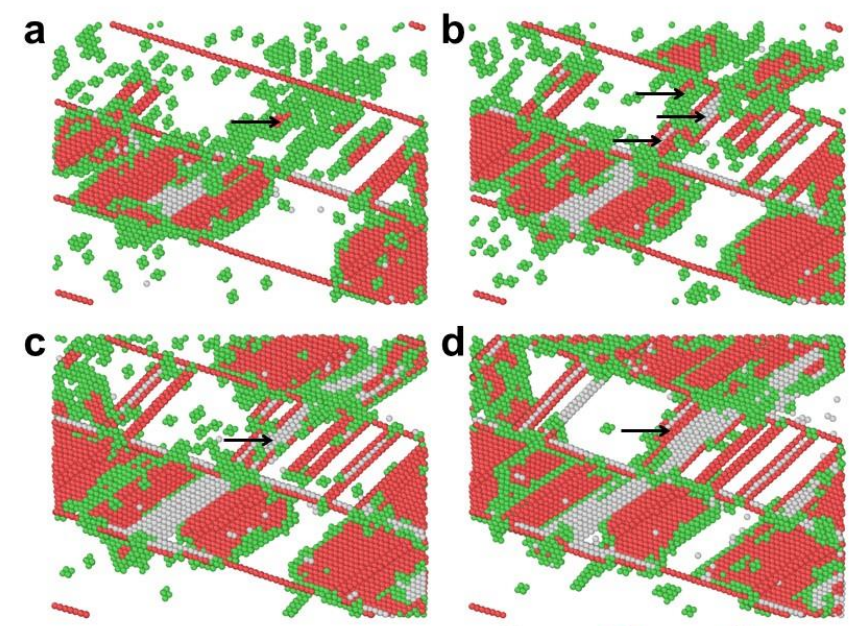

fcc $\bigcirc$ hcp other

Fig. 5. The formation of deformation twins through the agglomeration of twin nuclei (Region A2 in Fig. 3). (a) Dislocation nucleation at $8.73 \%$ strain. (b) The independent formation of three twin nuclei at $8.76 \%$ strain. (c) The agglomeration of two deformation twins at $8.79 \%$ strain. (d) Another combination at $8.83 \%$ strain.
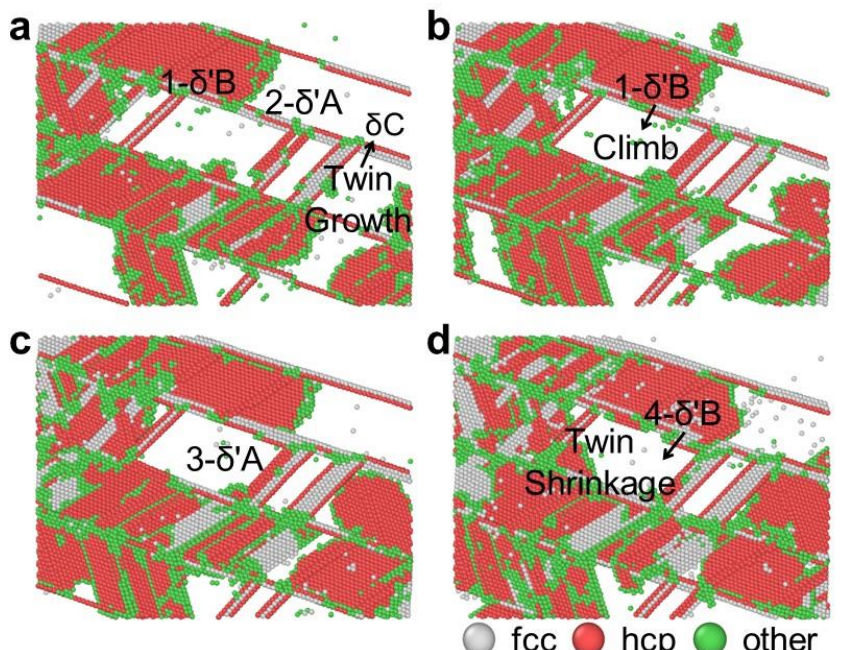

Fig. 6. The shrinkage of original growth twins through the glide of dislocation of different types (Region A3 in Fig. 3). (a) The motion of two partial dislocations reduces the twin thickness at $8.79 \%$ strain. (b) The first partial climbs downwards and its slip further thins the growth twin at $8.85 \%$ strain. (c-d) Further shrinkage of the growth twins at $8.89 \%$ strain (c) and at $8.99 \%$ strain (d). 


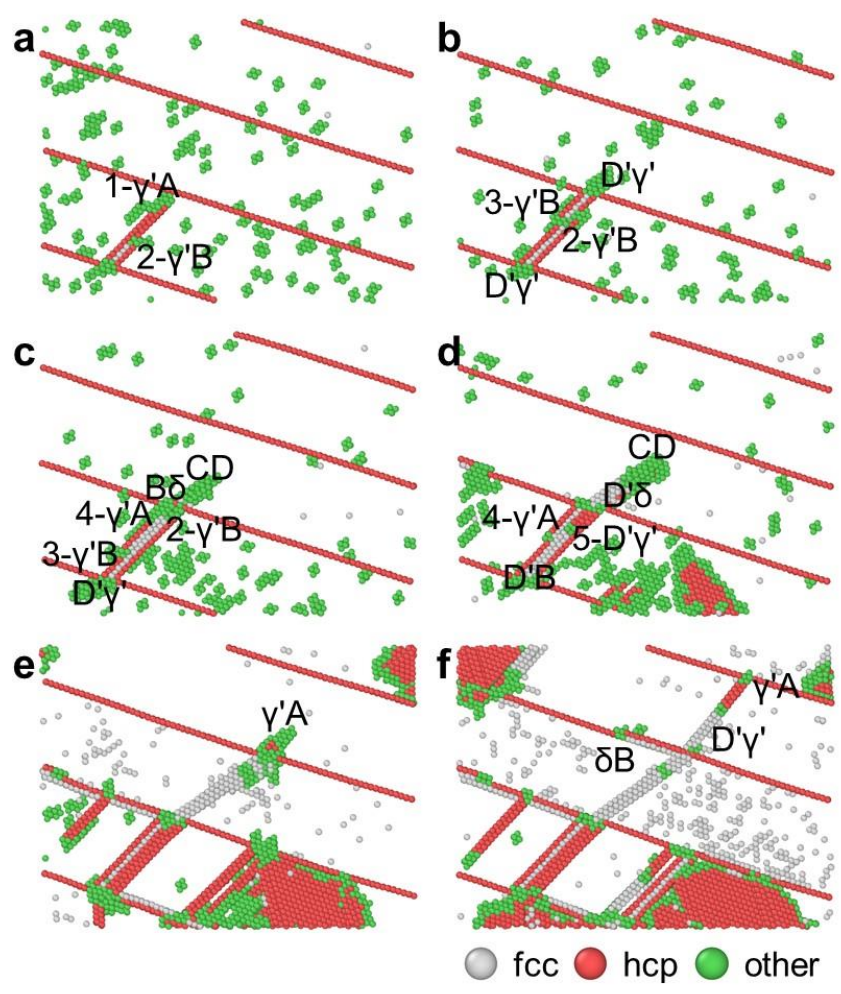

Fig. 7. Slip-twin interactions in sample T4A19.5 (Region A4 in Fig. 3). (a) The deposition of a double-Shockley partial on the TB at $8.7 \%$ strain. (b) Another deposition and the formation of a three-layer twin at $8.76 \%$ strain. (c) Nucleation of another twinning dislocation to form a full dislocation, which transmits across the TB to emit a Lomer dislocation at $8.79 \%$ strain. (d) The motion of Lomer dislocation and the nucleation of a trailing dislocation at $8.83 \%$ strain. (e) The transmission of the Lomer dislocation to emit a leading partial at $8.93 \%$ strain. (f) The full transformation of Lomer dislocation to form an extended dislocation at $9.22 \%$ strain. 

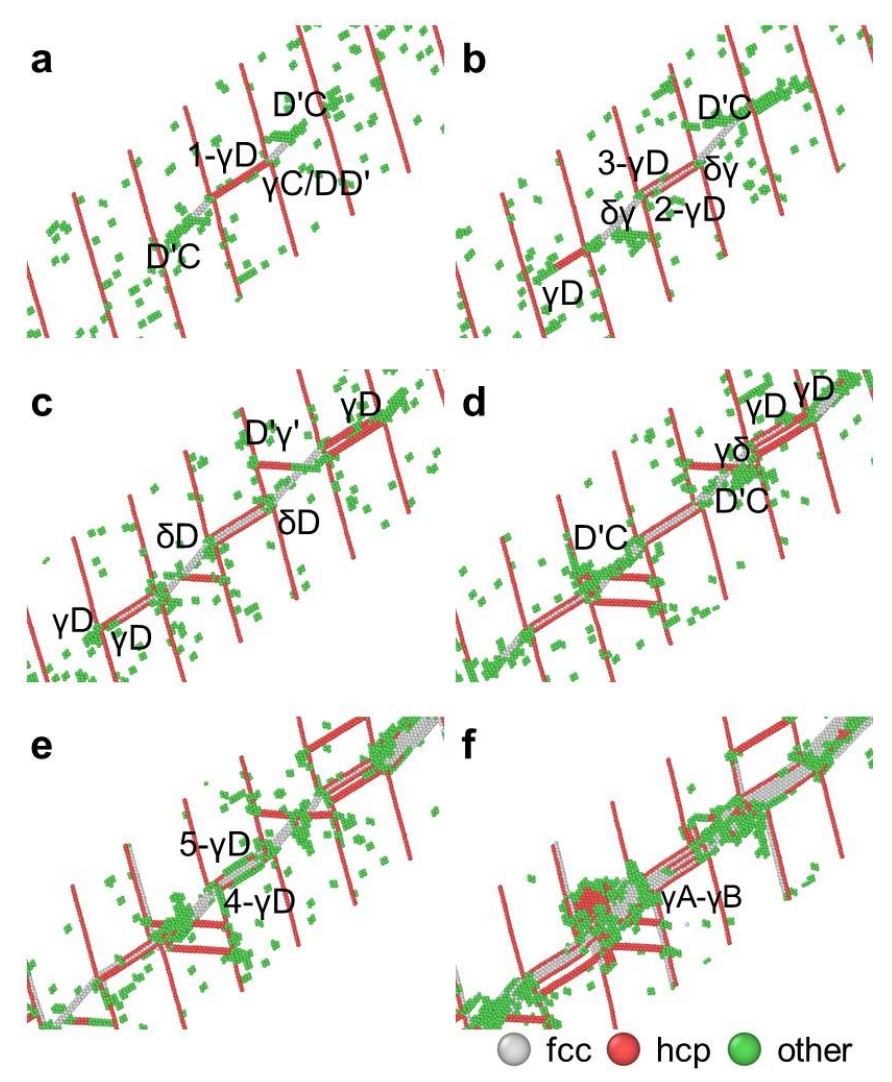

0.

1. $A B C A B C \stackrel{\triangle B A C A}{ } 1-\gamma D(a)$

2. $A B C A B C B A B C A B$ 2- $Y D$ (b)

3. CABCACBABCAB 3- $ү D(\mathrm{c} / \mathrm{d})$

4. CABCACBACABC 4-үD (e)

5. BCABACBACABC 5-үD (e)

6. ABCBACBACABC 6- $\mathrm{YD}$

Fig. 8. Deformation twinning and dislocation-TB interactions in sample T4A75 (Region B in Fig. 3). (a) The transmission of a Shockley partial across the TB to emit Lomer dislocations in the twinned grains at $6.4 \%$ strain. (b) The emission of two more Shockley partials and their combinations with the residual dislocations on the TBs at $6.45 \%$ strain. (c) The formation of Frank dislocations and the dissociation of a Lomer dislocation at $6.5 \%$ strain. (d) The transmission of Lomer dislocations at $6.56 \%$ strain. (e) Further growth of deformation twins at $6.63 \%$ strain. (f) The formation of a shear band from complicated dislocation-TB interactions at $6.8 \%$ strain. The stacking sequences during deformation are shown below the figure. The red letters indicate TB and the twin lamella are highlighted by grey colour. 


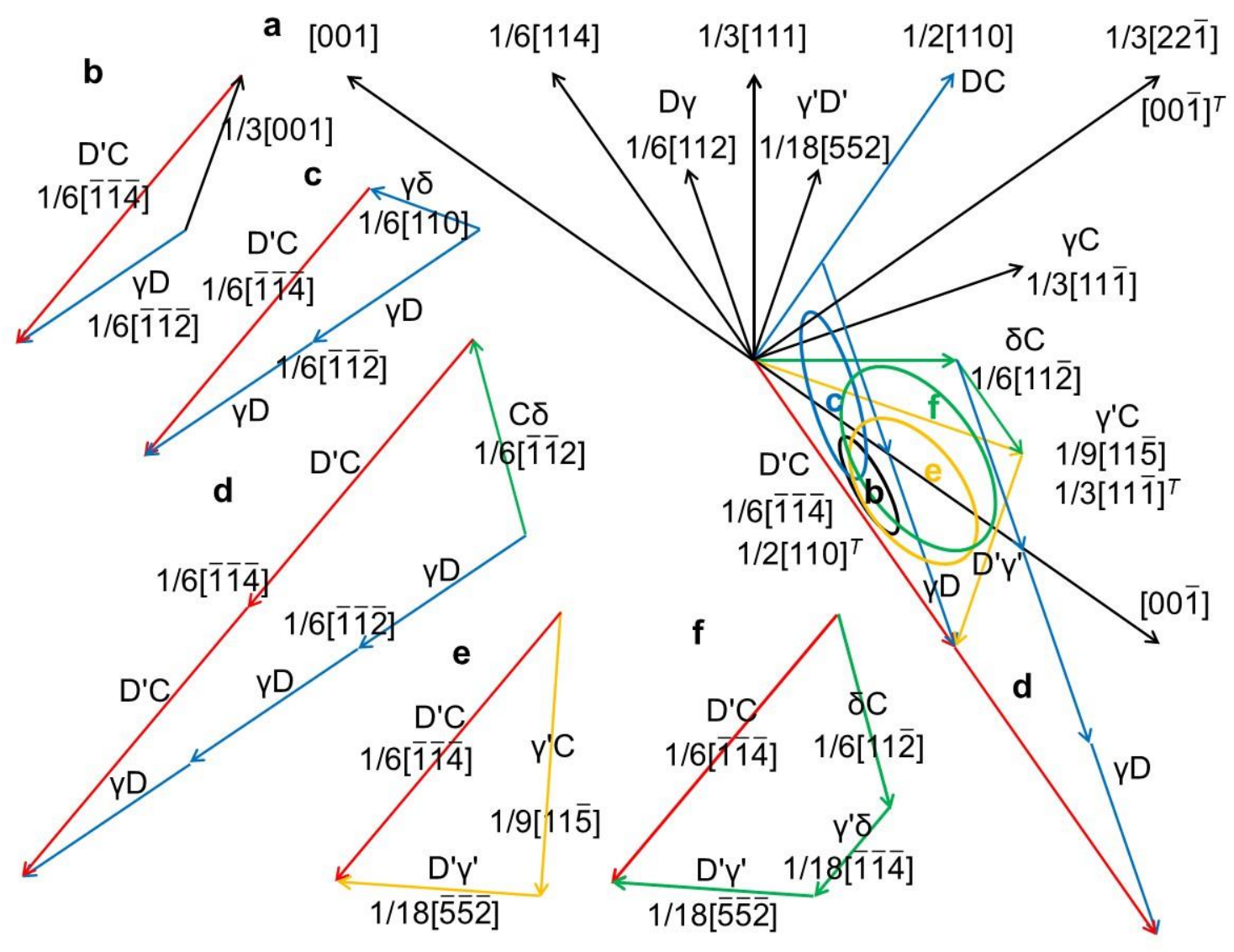

Fig. 9. Schematic illustration of slip directions and dislocation reaction diagrams. (a) Slip directions on (1 $1 \overline{1} 0)$ plane. (b-d) Dislocation reaction diagrams for the transmission of one (b), two (c) and three (d) twinning dislocations. (e-f) Dislocation reactions diagram for the dissociation of Lomer dislocation. 

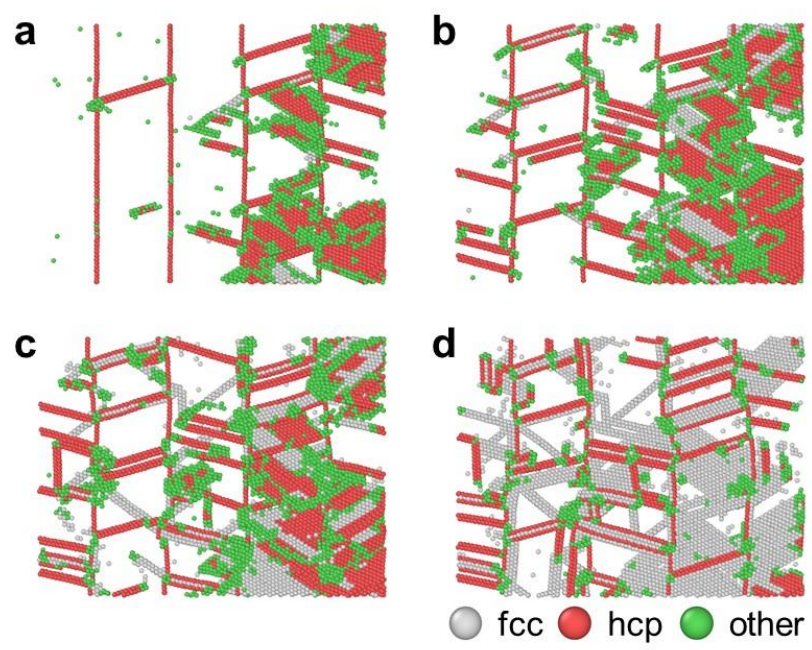

Fig. 10. Deformation twinning and dislocation-TB interactions in sample T4A90 (Region C in Fig. 3). (a) $8.3 \%$ strain. (b) $8.4 \%$ strain. (c) $8.5 \%$ strain. (d) $9 \%$ strain. 